\title{
Über den Einfluß des Lichtes auf die Färbung und die Entwicklung von Eiern von Asterias in Lösungen ver- schiedener Farbstoffe.
}

\author{
Von \\ Leo Loeb. \\ Aus dem patholog. Laboratorium der University of Pennsylvania, Philadelphia, \\ und ans dem Marine Biological Laboratory, Woods Hole, Mass.
}

Eingegangen am 8. Dezember 1906.

Durch die Untersuchungen einer Reihe von Forschern (RAAB, v. Tappeiner und Jodlbauer, Ledoux-Lebard, Straub, Edlefsen und andre 1)) wurde festgestellt, daß fluorescierende Stoffe, wie z. B. Eosin, auf Zellen und gewisse Fermente im Lichte eine viel schädlichere Wirkung austiben als im Dunkeln.

Im folgenden sollen einige Befunde mitgeteilt werden, die an Zellen (Seesterneiern) erhoben wurden, welche in Mischungen ver-

1) Es seien hier nur einige Veröffentlichungen angeführt: 1) Über die Beteiligung des Sauerstoffs bei der photodynamischen Wirkung fluorescierender Stoffe. A. Jodlbauer u. H. v. Tappeiner, Münchn. Med. Wochenschr. 28. Juni 1904. Nr. 26. 2) Über chemische Vorgänge bei der Einwirkung von Licht auf fluorescierende Substanzen (Eosin und Chinin) und die Bedeutung dieser Vorgänge für die Giftwirkung. W. Straub, Münchn. Med. Wochenschr. Nr. 25. 1904. 3) Experimenteller Beitrag zum Studium der oxydierenden Wirkung flnorescierender Stoffe. Eolersen, Münchn. Med. Wochenschr. Nr. 36. 1904. 4) Weitere Untersuchungen iber die Einwirkung des Sonnenlichtes auf fluorescierende Substanzen. Münchn. Med. Wochenschr. Nr. 41. 1905. 5) Ledoux-Lebard, Action de la lnmière sur la toxicité de l'éosine et de quelques autres substances pour les paramécies. Annales de l'Institut Pasteur. Vol. 16. 1902.

Nach Abschluß dieser Arbeit - der letzte Versuch wurde Ende August angestellt - erschien eine Mitteilung von V. SCHLÄPFER (PFLÜGERs Archiv. Bd. 114. Heft 7/8). Die Ergebnisse dieses Autors haben viele Berïhrungspunkte mit den hier mitgeteilten.

Archiv f. Entwicklungsmechanik. XXIII. 
schiedener Farblösungen gehalten wurden. Es sollte geprüft werden, ob sogenannte saure und basische Farbstoffe in bezug auf ihre Wirkungen auf Zellen eine antagonistische Wirkung aufeinander austiben, oder ob die Wirkung des Lichtes in solchen Mischungen ungeschwächt vorhanden ist, so daß eine additive Wirkung beider Komponenten der Farbstoffmischung vorliegt. Bei Gebrauch von Vitalfarbstoffen sollten Unterschiede in der Färbung. im Dunkeln und im Lichte einen Schluß anf Beeinflussung der Zellen durch das Licht zulassen. Sollten sich Unterschiede in der Färbung der Zellen im Lichte und im Dunkeln finden, so wäre weiter zu untersuchen, ob es möglich ist, diese Unterschiede experimentell zu vergrößern oder zu verringern, um womöglich Einsicht in die Wirkungsweise des Lichtes zu gewinnen. Mit dem Einfluß des Lichtes auf die Färbung sollte der Einfluß auf die Entwicklung der Eier verglichen werden. Letzteres war nur in unvollständiger Weise möglich, da die Seesterneier, die zu diesen Versuchen dienten, sich sehr oft nur in ungenügender Weise entwickelten. Diese und einige andre Fragen sollen sobald wio möglich an neuem Material wieder aufgenommen werden.

$\mathrm{Zu}$ diesen Versuchen wurden vor allem Kombinationen von Methylenblau und Eosin, sowie von Neatralrot und Eosin benutzt; es wurden aber auch Versuchsserien mit Säurefuchsin und Methylenblau, Süurefuchsin und Neutralrot, Eosin und Methylviolett, Eosin und Hämatoxylin, Säurefuchsin und Hämatoxylin, und Methylenblau und Nentralrot ausgeführt. Zu jeder Versuchsreihe wurden die Farbstoffe jeweils in verschiedenen Proportionen gemischt. Daneben wurden in jedem Experiment die Komponenten der Farbstoffgemische allein in verschiedenen Verdünnungen geprüft, auch wurden Kontrollversuche mit Seewasser im Lichte und im Dunkeln angestellt. Die Farbstoffe wurden in Seewasser gelöst, in dem sie mit Ausnahme von Neutralrot, das gewöhnlich ein Präcipitat von kristallinischen Nadeln ausfallen ließ, sich leicht lösten. Zur Herstellung der Mischungen wurde $1 \mathrm{~g}$ Eosin in $5000 \mathrm{cem}$ Seewasser gelöst. Dieselbe Lösung wurde von Säurefuchsin hergestellt. Methylenblau, Methylviolett, Hämaltoxylin, Neutralrot wurden in dem Verhältnis 1: 10000 gelöst. Auch wurden Neutralrotlösungen 1:5000 benutzt. Folgende Mischungen wurden gewöhnlich hergestellt: a) 20 Teile Lösung des sauren Farbstoffs +1 Teil Lösung des basischen Farbstoffs. b) 10 Teile Lösung des sauren Farbstoffs +1 Teil Lösung des basischen Farbstoffs. c) 5 Teile Lösung des sauren Farbstoffs +1 Teil Lösung des basischen Farbstoffs. d) Gleiche Teile der basischen und sauren Farb- 
stofflösung. e) 1 Teil der Lösung dès sauren Farbstoff́s +2 Teilè Lösung des basischen, Farbstoffs. Bei Mischung von Nentralrot und Methyleublau wurde Nentralrot in den Proportionen des Eosin angewandt.

Je 50 cem der Farbmischung oder der Komponenten der Farbmischung sowie des. Seewassers wurden in eine Glasschale gebracht und sodann die Eier bald nach der Befruchtung. oder mehrere Stunden später der Lösung zugesetzt. Die Schalen wurden mit Glasplatten bedeckt. , Eine Schale stand im diffusen Tageslicht, eine andre mit derselben Lösang stand unter im übrigen gleichen Bedingungen im Dunkeln. Nur wenige Male wurde Sonnenlicht benutzt. In der großen Mehrzahl der Versuche wurden die Eier 2 Tage lang beobachtet, in einigen Fällen war die Beobachtungszeit länger. Die folgenden Befunde wurden erhoben:

1) Kombination von Eosin und Methylenblau. Mit diesen beiden Farbstoffen wurde eine große Anzahl von Versuchsreihen angestellt. Im wesentlichen war das Resultat in den einzelnen Versuchen das gleiche. In Kontrollversuchen mit Seewasser allein ergab sich niemals ein bedeutender Unterschied zwischen den im Licht und im Dunkel gehaltenen Zellen. Falls Unterschiede bestehen, miissen dieselben geringfügiger Natur sein. Erst weitere Untersuchungen können darüber Aufschluß geben, besonders weil Fäulnis der toten Eier die Versuchsergebnisse nicht selten kompliziert. In Lösungen von Eosin allein ist ein deutlicher Unterschied zwischen den im Lichte und im Dunkeln gehaltenen Eiern vorhanden. Die Entwicklang der Eier ist besser im Dunkeln als im Lichte; die Zahl-der sich entwickelnden Blastulae und Gastrulae ist größer: im Dunkeln wie im Hellen. Im Lichte ist die Zahl der mit Eosin gefärbten Zellen etwas größer wie im Dunkeln. Es färben sich besonders die Zellen, deren Membran stark abgehoben ist, sowie solche Eier, die sich in mehrere Segmente geteilt haben, während, wenigstens am ersten Tage, auch im Lichte viele ungeteilte Eier mit bläschenförmigem Kern und weniger stark abgehobener Membran ungefärbt sind. Doch finden sich gewöhnlich auch viele Eier mit abgehobener Membran, die keine Farbe aufgenommen haben. Zuerst färbt sich gewöhnlich granuläres, aus den Eiern ausgeflossenes Cytoplasma, das sich unter der Eimembran ansammelt, mit Eosin. Später können sich dann auch Zellsegmente oder ungefurchte Eier färben. Je nach der Intensität des Lichtes und der Beschaffenheit der Eier finden sich in einzelnen Versuchen einige quantitative Unterschiede in bezug auf Färbung und Entwick- 
lung der Eier. Insbesondere können am zweiten Versuchstage die Unterschiede zwischen Licht und Dunkel größer sein wie am ersten; aber als ein wesentlicher Befund ergab sich die Tatsache, daß Eosin allein viel geringere Unterschiede in der Färbung der Eier im Lichte nnd im Dunkeln bedingt als eine Eosin-Methylenblaumischang, auch wenn diese nur eine geringe Menge Methylenblan enthält. Kleine Variationen quantitativer Natur, die sich in den einzelnen Versuchen finden, ändern an dieser Tatsache nichts. In den Eosin-Methylenblaumischungen $a, b$ und $c$ ist der Unterschied in der Färbung im Hellen und Dunkeln außerordentlich viel markanter, wie in Eosin allein.

Im Dunkeln sind die Unterschiede zwischen Losungen, die nur Eosin und solchen, die Eosin und Methylenblau (Mischung a) enthalten, gering. In Mischung a von Eosin und Methylenblau mag im Dunkeln eine geringe Zahl von Eiern stärker mit Eosin gefärbt sein, als in reinem Eosin, aber der Unterschied kann fehlen oder ist nur sehr geringfügig. Im Dunkeln kann ferner eine geringere oder größere Anzahl von Eiern sich schwach mit Methylenblau färben, während im Lichte diese Färbung fehlt oder merklich schwächer ist wie im Dunkeln. An der Aufnahme des Methylenblaus beteiligen sich sowohl ungefurchte Zellen wie Blastulae und Gastrulae.

Der wesentliche Unterschied in der Färbung der in reinem Eosin und in Mischung a von Eosin und Methylenblau gehaltenen Zellen zeigt sich aber erst in den im Lichte gehaltenen Eiern. Der Zusatz von ein wenig Methylenblau zu Eosin im Lichte bewirkt, daß gewöhnlich schon am ersten Tage die große Mehrzahl der Eier sich mit Eosin färbt, so daß der Unterschied in der Färbung von Eosin-Methylenblau a im Lichte und Dunkel viel deutlicher ist, wie der Unterschied in der Färbung der entsprechenden Zellen in Eosin allein. Das gleiche gilt für die Mischungen $b$ und $c$ von Methylenblau-Eosin. Der Zusatz von ein wenig Methylenblau verstärkt also bedeutend die Eosinfärbung der Zellen im Lichte.

Weiterhin ist Zusatz von Methylenblau zu Eosin ungünstig für die Entwicklung der Eier; in diesen Mischungen entwickeln sich weniger Eier zu Blastulae und Gastrulae wie in Eosin allein. Auch für die Entwicklung der in Eosin-Methylenblau gehaltenen Eier ist das Licht schädlich. Im Lichte entwickeln sich die Eier weniger gut wie im Dunkeln.

Doch übt auch auf die im Dunkeln gehaltenen Eier Zusatz von 
Methylenblau einen entwicklungshemmenden Einfluß aus, so daß in den Eosin-Methylenblaulösungen die Zahl der Blastulae und Gastrulae gewöhnlich geringer ist wie in den reinen Eosinlösungen.

In den Mischungen $d$ und $e$, die eine größere Menge Methylenblau enthielten, nahmen die Eier im Lichte eine Mischfarbe zwischen Rot und Blau an, sie färbten sich purpurn, im Dunkeln hingegen war die blaue Komponente stärker vertreten, so daß die Eier sich mehr oder weniger rein blau färbten. In Mischung e war die blaue Komponente relativ stärker vertreten in der Färbung der Eier, und zwar gilt dies sowohl für die im Lichte wie für die im Dunkeln gehaltenen Eier; dies entspricht der größeren Menge Methylenblan, die in Mischung e enthalten war.

In Mischungen d und e entwickeln sich die Eier nicht zu Blastulae, da Methylenblau bedeutend giftiger auf die Eier wirkt wie Eosin.

Die schädliche Wirkung des Eosin in bezug auf die Entwicklung und Lebensdauer der Eier ist um so stärker, je konzentrierter die Eosinlösung ist. Eine Lösung 1:5000 wirkt stärker als 20000 fach verdünntes Eosin. Wahrscheinlich wirkt Eosin schädlicher anf Blastulae wie auf Bipinnarien, die eine höhere Entwicklungsstufe der Seesterneier darstellen. Bipinnarien lebten in zwei Versuchen in der Eosinlösung 3 Tage. Doch war auch hier der schädliche Einfluß des Lichtes sehr deutlich.

Methylenblau allein ist für die Eier viel giftiger wie Eosin. Methylenblau 1:10000 verhindert sowohl im Lichte wie im Dunkeln die Entwicklung der Eier zu Blastulae und Gastrulae. In Methylenblaulösungen 1:100000 können sich jedoch diese Entwicklungsstufen finden. In den reinen Methylenblaulösungen färben sich die Zellen blau. Die im Dunkeln gehaltenen Eier sind gewöhnlich ein wenig: intensiver blau gefärbt wie die dem Lichte exponierten Zellen. Auch in beweglichen Blastulae oder Gastrulae können sich Zellkomplexe diffus blau färben. Aber der Unterschied in der Färbung der Zellen im Lichte und im Dunkeln ist in reinen Methylenblaulösungen gewöhnlich nicht sehr groß und dieser Unterschied entspricht, wie wir später sehen werden, wahrscheinlich einem geringen Unterschied in der Färbung der Lösung, indem im Lichte die Lösung ein wenig gebleicht wird.

2) Kombination von Eosin und Methylviolett. In dieser Mischung, mit der bisher nur zwei Versuche angestellt werden konnten, trat das Methylviolett an die Stelle von Methylenblau, und es wurde in denselben Proportionen angewandt wie Methylenblau. 
In den Mischungen $\mathfrak{a}$, b und c war der Untersehied zwischen Licht und Dunkel nur geringfugig. Die Zellen waren entweder ungefärbt oder hatten eine Rosa- bis schwach Purpur-Farbe: Also bei Zusatz von relativ geringen Mengen von Methylviolett hatte dieses nicht die Wirkung von Methylenblau, indem es nicht die Eosinfärbung in der Weise verstärkte, wie das Methylenblau es tut. Wohl aber war bei Zusatz von größeren Mengen Methylviolett (Mischungen $d$ und e) der Unterschied in der Färbung zwischen Licht und Dunkel sehr ausgeprägt. Die im Dunkeln gehaltenen Zellen waren hier blau bis violett gefärbt, im Hellen nahmen die Zellen eine rote oder rotpurpurne Farbe an. Auch die Lösungen selbst zeigten gewisse Unterschiede in der Färbung, im Hellen war eine rötliche, im Dunkeln eine violette Nuance vorhanden. In dem reinen Methylviolett (1:10000) waren die Zellen im Hellen und Dunkel violett gefärbt; doch war die Farbe im Dunkeln etwas intensiver wie im Lichte, entsprechend der etwas tieferen Färbung, welche die Methylviolettlösung im Dunkeln zeigte. Auch bei stärkerer Verdünnung des reinen Methylvioletts (1: 100000) waren die Zellen im Dunkeln etwas mehr bläulich gefärbt wie im Lichte.

3) Kombination von Neutralrot und Eosin. In dieser Mischang vertrat Neutralrot das Methylenblau; das Neutralrot wurde in Verdünnungen 1:5000 und 1:10000 verwendet. In einer größeren Anzahl von Versuchen ergab sich, daß sowohl in reinen Neutralrotlösungen wie in Kombinationen von Nentralrot und Eosin die Färbung der im Lichte und im Dunkel gehaltenen Eier verschieden ist. Im Dunkeln färben sich die Eier stärker mit Neutralrot als im Lichte. In den Mischungen von Eosin und Neutralrot nehmen die im Hellen gehaltenen Zellen mehr Eosin und weniger Neutralrot auf als die im Dunkeln gehaltenen Eier. Dieser Unterschied in dem Verhalten der Eier tritt besonders klar hervor, wenn wir die Farbe der Lösungen mit der Farbe der Eier vergleichen. Während im Dunkeln am ersten und am zweiten Tag die Eier sich deutlich mehr mit Neutralrot färben als im Lichte, ist umgekehrt die Farblösung selbst am ersten Tag im Lichte stärker gefärbt wie im Dunkeln, wahrscheinlich, weil im Dunkeln die Eier der Lösung mehr Neutralrot entziehen wie im Lichte. Am zweiten Tage ist dieser Unterschied in der Färbung der Lösung verschwunden, obwohl die Eier gewöhnlich noch Unterschiede in der Färbung zeigen. Dies beruht wahrscheinlich darauf, daß im Lichte die Lösung allmählich stärker entfärbt wird wie im Dunkeln. Doch auch die Eier werden bei längerem Stehen mehr oder weniger 
entfärbt; dieselben haben am zweiten Tage weniger Farbe wie am ersten und zwar findet diese Entfärbung sowohl im Hellen wie im Dunkeln statt. Am ersten Tage ist im Dunkeln die Zahl der Eier, welche kirschrot bis dunkelrot oder dunkelbraunrot gefärbt sind, viel größer wie im Lichte. Im Hellen sind viele Eier gelb bis gelbbraun gefärbt oder ganz ungefärbt. Im Dunkeln ist 1) die Zahl der Eier, die mit Neutralrot gefärbt sind, größer als im Lichte, 2) die Intensität der Färbung der einzelnen Zellen ist im Dunkeln größer als im Lichte. Doch ist auch im Hellen gewöhnlich eine gewisse Anzahl der Eier rot bis braunrot gefärbt. Im übrigen finden wir Unterschiede in der Färbung der verschiedenen Eier je nach der Entwicklungsstufe, auf der sie sich befinden. Am tiefsten färben sich lebende Blastulae und Gastrulae, sie nehmen eine dunkelbraunrote oder violette Farbe an und zwar ist die Färbung gewöhnlich so tief, daß die feinere Struktur der Organismen nicht erkennbar ist. Die nicht segmentierten Eier färben sich im Dunkeln sehr oft kirschrot bis weinrot. Der Kern bleibt immer ungefärbt; doch können sich die unsegmentierten Eier auch dunkler färben. Die zwischen ungeteiltem Ei und Blastula stehenden Entwicklungsstufen färben sich gewöhnlich relativ am wenigsten; sie färben sich entweder heller rot, oder haben eine gelbbraune Färbung, oder sie sind ungefärbt. Auch im Dunkeln färben sich diese segmentierten Eier relativ weniger wie Eier, die entweder ungeteilt oder zur Blastula entwickelt sind.

Die Intensität der Neutralrot-Färbung der einzelnen Zellen hängt nun auch von den Proportionen ab, in denen Eosin und Neutralrot gemischt sind. Je mehr Neutralrot in den Mischungen vorhanden ist, desto intensiver färben sich die Zellen im Dunkeln und im Hellen mit Neutralrot; je mehr Eosin vorhanden ist, desto weniger färben sich die Zellen im Dunkeln sowohl wie im Hellen mit Neutralrot. So färben sich zum Beispiel in Mischung a im Dunkeln die unsegmentierten Eier nur ganz schwach mit Neutralrot. In Mischungen, die mehr Neutralrot enthalten, färben sie sich intensiver rot. In bezug auf die Färbung mit Eosin liegen in den Neutralrot-Eosinmischungen ähnliche Verhältnisse vor wie in den Methylenblau-Eosinmischungen, insofern als auch hier im Lichte die Zellen sich viel stärker mit Eosin färben wie im Dunkeln. Es scheint, daß auch hier in Mischung a die Zellen im Lichte mehr Eosin aufnehmen wie in Eosin allein. Der Zusatz von Neutralrot würde also in bezug auf die Färbung mit Eosin im Lichte ähnlich wirken wie der Zusatz von ein wenig Methylenblan zu Lösungen von Eosin. Doch ist die 
Schwierigkeit in der Feststellung dieser Tatsache bei Anwendung der Eosin-Neutralrotfärbung größer wie im Falle der Eosin-Methylenblanmischung, da bei Gebrauch der letzteren Mischung das Blau des Methylenblaus einen stärkeren Kontrast zu dem Rot des Eosins bildet als das Neutralrot.

Der Unterschied in der Färbung im Lichte und im Dunkeln beruht 1) darauf, daß Zellen, die anscheinend die gleiche Vitalität besitzen, z. B. bewegliche Blastulae und Gastrulae, im Dunkeln stärker mit Nentralrot gefärbt sind wie im Lichte, oder indem wohl erhaltene unsegmentierte Eier mit normalem bläschenförmigem Kern im Dunkeln sich stärker mit Neutralrot färben wie im Lichte. Es scheint aber auch, daß 2) das Licht die Eier selbst verändert, da die Zahl der Eier mit weit abgehobener Membran im Lichte größer ist als im Dunkeln. Eier mit weit abgehobener Membran färben sich nun gewöhnlich gelbbraun. Die Färbung im Lichte mit Neutralrot ist also derart, als ob die Zellen großenteils eine schwach alkalische Reaktion besäßen, während sie sich im Dunkeln vielfach so färben, als ob sie schwach sauer oder neutral reagierten.

In den Mischungen, welche Eosin enthielten, war die Entwicklung der Eier im Lichte weniger gut wie im Dunkeln. Aber auch in Neutralrot allein war gewöhnlich die Entwicklung besser im Dunkeln wie im Hellen. Ob dies ein konstanter Befund ist, soll später womöglich mit besserem Material weiter untersucht werden.

Zusatz von Neutralrot zu Eosin ist für das Leben und die Entwicklung der Eier bedeutend weniger schädlich wie Zusatz von Methylenblau zu Eosin.

4) Kombination von Säurefuchsin und Neutralrot. In dieser Mischung trat Säurefuchsin an die Stelle von Eosin. Säurefuchsin färbt die Eier gar nicht oder nur in außerordentlich geringem Maße; es findet sich zuweilen eine Spur von Rosafärbung. In dieser Mischung handelt es sich daher im wesentlichen um eine Neutralrotfärbung der Eier und was oben über die Färbung der Zellen mit Neutralrot im Hellen und im Dunkeln gesagt wurde, gilt in der Hauptsache hier. Die Anwesenheit von Säurefuchsin in der Mischung. ändert nichts wesentliches an der Einwirkung des Lichtes auf die Färbung mit Neutralrot.

Auch hier war die Färbung der Zellen mit Neutralrot um so intensiver, je mehr Neutralrot in der Mischung vorhanden war. In gleicher Weise war auch hier die Entwicklung der Eier gewöhnlich besser im Dunkeln wie im Lichte. 
5) Kombination von Säurefuchsin und Methylenblau. In Mischungen $a, b$ und $c$ sind die Zellen im Dunkeln blau gefärbt; doch bleibt auch hier eine gewisse Zahl von Eiern ungefärbt; im Hellen ist die Farbe der Zellen viel weniger intensiv blau; sehr viele Zellen sind ungefärbt. Im Lichte ist die Farbe der Lösung mehr oder weniger grünblau, im Dunkeln ist sie mehr reinblau.

In Mischungen $d$ und e liegen die Verhältnisse ähnlich, doch ist hier die blaue Färbung relativ stärker, im Lichte sowohl wie im Dunkel. Also im Gegensatz zu Eosin färbt Säurefuchsin auch die toten Zellen fast gar nicht in den Mischungen. Schwimmende Blastulae kommen in der Mehrzahl der Mischungen nicht zur Entwicklung.

Methylenblau ist giftiger als Säurefuchsin und Zusatz von Methylenblau zu Säurefuchsin verschlechtert die Entwicklung der Eier. In Säurefuchsin allein ist daher die Entwicklung der Eier besser als in den Mischungen mit Methylenblau. In Säurefuchsin allein färben sich die Zellen nur sehr wenig; sie können sich ganz schwach gelb bis rosa färben, und zwar gelegentlich im Dunkeln etwas mehr als im Hellen, aber auch im Dunkeln ist die Färbung nur sehr gering.

6) Kombination von Säurefuchsin und Hämatoxylin. In dieser Kombination vertrat das Hämatoxylin die Stelle des Methylenblaus oder des Neutralrots. Die Zellen waren sowoll im Lichte wie im Dunkeln fast ganz ungefärbt, ein merklicher Unterschied in der Färbung der Zellen im Hellen und im Dunkeln bestand nicht. Doch nahmen manche Zellen, besonders segmentierte Eier, einen braunen Ton an und zwar vielleicht im Dunkeln ein wenig mehr wie im Lichte; diese Braunfärbung war besonders merklich in den Lösungen, die Hämatoxylin in stärkeren Proportionen enthielten.

Diese Kombination war für die Entwicklung der Zellen nicht ungiunstig; ein wesentlicher Unterschied zwischen Hell und Dunkel bestand jedoch in bezug auf die Entwicklung der Eier nicht in diesen Lösungen.

7) Kombination von Eosin und Hämatoxylin. Hämatoxylin allein färbt die Zellen nicht oder nur sehr wenig und zwar braun; es werden hauptsächlich die toten Zellen gefärbt. In Kombination mit Eosin hat Hämatoxylin nicht die volle Wirkung des Methylenblaus; es verstärkt nämlich nicht wesentlich die Färbung der Zellen mit Eosin im Lichte; jedenfalls ist es in den Proportionen, in denen Methylenblau wirksam ist, in dieser Hinsicht unwirksam.

Ein Zusatz von Hämatoxylin zu Eosin scheint günstig auf die Entwicklung der Eier zu wirken; besonders in den Mischungen b 
und c blieben die Eier länger am Leben wie in Eosin allein. Auch in diesen Mischungen nehmen die Zellen oft einen braunen Ton an, und zwar ist diese Färbung im Dunkeln etwas stärker als im Lichte; doch ist dieser Unterschied nicht sehr groß und hängt vielleicht mit der oft im Dunkeln etwas stärkeren Färbung der Lösung selbst zusammen.

In Hämatoxylinlösungen $(1: 100000)$ können Bipinnarien tagelang am Leben bleiben. In dieser Lösung schrumpfen die lebenden Organismen beträchtlich.

8) Kombination von Methylenblau und Neutralrot. In dieser Kombination, mit der bisher nur eine Versuchsreihe angestellt werden konnte, liegt eine Mischung zweier basischer Farbstoffe vor. Neutralrot $(1: 5000)$ wurde in den Proportionen des Eosin, Methylenblau in den gewöhnlichen Verhältnissen der basischen Farbstoffe zugesetzt.

Die Farbe der Lösungen ist hier versehieden in Dunkeln und im Lichte. Im allgemeinen überwiegt in den Mischungen die Methylenblaufarbe. In denjenigen Lösungen, die viel Nentralrot enthalten, tritt die Nentralrotkomponente im Dunkeln stärker als im Hellen hervor. In Mischung a ist z. B. am ersten Tag die Farbe der Fliussigkeit in Lichte gelblichgrün, im Dunkeln hingegen überwiegt die rote Komponente; am zweiten Tage war die Farbe im Lichte grün, in Dunkeln hellorange. Auch in Lösung $\mathrm{b}$ tritt im Dunkeln die braune Komponente des Neutralrots stärker hervor, während im Lichte die Farbe ein reineres Grün ist. In solchen Mischungen, die noch mehr Methylenblau enthielten, war die Farbe grün oder grünlichblau und der Unterschied in der Färbung der Flüssigkeiten im Hellen und im Dunkeln war nur gering.

Die Unterschiede in der Färbung der Zellen im Lichte und im Dunkeln waren sehr markant. In Mischung a färbten sie sich im Lichte am ersten Tage gelbgrün und nur wenige Zellen färbten sich dunkelbraun; im Dunkeln hingegen färbten sich die Segmente der gefurchten Eier und die ungeteilten Eier dunkelbraun. Im Dunkeln nehmen also die Zellen Neutralrot auf - was den oben mitgeteilten Tatsachen über den Einfluß des Lichtes auf die Neutralrotfärbung der Zellen entspricht - ; im Lichte färben sich die Zellen mit einer Mischfarbe von Neutralrot and Methylenblau; hierbei überwiegt jedoch das Methylenblau und zwar besonders am zweiten Tage. In Mischungen $\mathrm{b}$ und $\mathrm{c}$ bestehen ähnliche Unterschiede in der Färbung der Zellen im Lichte und in Dunkel wie in Mischung a, mit der Abweichung, 
daß die Methylenblankomponente der Zellfärbung im Lichte : noch stärker wird und daß am zweiten Tag die Methylenblaukomponente stärker hervortritt und daß zu dieser Zeit die Neutralrotfärbung sogar im Dunkeln schwindet. In Mischung e und in solchen Mischungen, die noch mehr Methylenblau enthalten als Mischung e, tritt im Dinkeln am ersten Tage fast eine reine Methylenblaufärbung der Zellen auf, im Lichte findet sich hingegen wiederum eine Mischfarbe, indem die Eier braunblau oder braungrün gefärbt sind. Wir sehen also, daß im Dunkeln entweder eine reine Neutralrot- oder eine reine Methylenblaufärbung der Zellen eintritt, je nach den Proportionen, in denen die beiden Farbstoffe in den Gemischen enthalten sind. - Im Lichte hingegen färben sich die Zellen mit einer Mischfarbe, wobei das Methylenblau iiberwiegt. Also Neutralrot und Methylenblau vertreten sich im Dunkeln; uberwiegt die Menge des Neutralrots sehr stark in der Mischung, so färben sich die Zellen mit Neutralrot, ist das Methylenblau in gewissen Proportionen vorbanden, so färben sich die Zellen mit Metbylenblau. Diese Ersetzbarkeit einer Farbe durch eine andre im Dunkeln ist nur möglich, da Methylenblau und Neutralrot beide vitale Färbungen ermöglichen; bei Kombination von Neutralrot oder Methylenblan mit dem sauren Farbstoff Eosin ist eine solche Ersetzbarkeit nicht vorhanden. Auch bei Anwendung von großen Mengen Eosin in den Gemischen färbten sich am ersten Tage im Dunkeln die Zellen nur sehr wenig, und zwar offenbar nur die toten Zellen.

Aus diesen Beobachtungen ergibt sich also, daB Zellen, je nachdem sie im Lichte oder im Dunkelngehalten werden, sich verschieden färben. Dies gilt insbesondere für Färbung mit Eosin, Methylenblau, Methylqiolett und Neutralrot, sowie für Kombinationen dieser Farbstoffe und vielleicht auch, wenn auch nur in geringem Maße, für Hämatoxylin. In bezug auf die Entwicklung der Eier ist es sicher, daß dieselbe merklich besser im Dunkeln als im Lichte in allen solchen Lösungen ist, welche Eosin enthalten. Es ist nicht unwahrscheinlich, daß das gleiche auch für Neutralrot gilt. Es ergab sich ferner, daß Zusatz einer geringen Menge Methylenblau zu Eosin den Unterschied in der Färbung der Eier im Lichte und im Dunkeln bedeutend verstärkt. Das Methylenblau neutralisiert also nicht nur nicht die Vorgänge, welche za einer Färbung der Zellen mit Eosin führen, sondern verstärkt sie im Gegenteil bedeutend, sobald die Zellen dem Lichte ausgesetzt werden. Es ist wahrscheinlich, daß auch Zusatz von Neutralrot zu Eosin die Färbung der Zellen mit Eosin beguinstigt. 
Im weiteren wird es sich nun darum handeln, die Wirkung des Lichtes auf die Färbung der Zellen weiter zu analysieren.

a) Wir sahen, daß die Lösungen selbst im Lichte hänfig eine andre Farbe annehmen wie im Dunkeln. Entspricht nun der Unterschied in der Färbung der Zellen den Unterschieden in der Färbung der Lösung? Das ist nicht der Fall in den Lösungen, welche Eosin oder Neutralrot allein oder in Kombination mit Methylenblan enthalten. Besonders deutlich tritt der Unterschied in der Färbung der Zellen und der Flüssigkeit in Neutralrotlösungen am ersten Tag hervor, indem im Dunkeln die Zellen stärker mit Neutralrot gefärbt sind als im Lichte, während die Flussigkeit umgekehrt stärker im Lichte gefärbt ist als im Dunkeln. Wir können also folgern, daß die Zellen nicht nur passiv im Lichte und im Dunkel die Unterschiede in der Färbung der im Dunkeln oder Licht gehaltenen Lösungen wiedergeben.

b) Es war nun von Interesse zu untersuchen, wie die Färbung toter Zellen durch das Licht beeinflußt wird. Die Zellen wurden daher durch kurzdanerndes Erwärmen auf $47-50^{\circ}$ abgetötet. Solche erwärmten Eier färbten sich in Lösungen, die Neutralrot enthielten, entweder leicht gelbbraun oder sie blieben ungefärbt; ein bedeutender Unterschied in der Färbung im Lichte und im Dunkeln bestand nicht. In solchen Fällen, in denen ein geringer Unterschied in der Färbung der Lösung bestand, konnte sich ein entsprechender geringer Unterschied in der Färbung der Eier im Lichte und im Dunkel zeigen.

In Eosin und in Mischung a von Eosin und Methylenblau färben sich die Zellen im Dunkeln und im Lichte in gleicher Weise stark mit Eosin.

In den Mischungen, die mehr Methylenblau enthalten und in reinen Methylenblaulösungen färben sich die Zellen im Dunkeln ein wenig mehr mit Methylenblau als im Hellen, entsprechend der Tatsache, daß die Methylenblaulösungen selbst im Dunkeln reiner und tiefer blau gefärbt sind als im Lichte. Wir sehen also, daß in durch Wärme getöteten Zellen die oben angegebenen Unterschiede in der Färbung im Lichte und im Dunkel zu einem großen Teil verschwinden; entweder färben sich die Zellen im Dunkeln und im Lichte gleich oder es bestehen die den Unterschieden in der Färbung der Flüssigkeiten selbst entsprechenden Unterschiede in der Färbung der Zellen. Hieraus folgt, daß die oben beschriebenen Unterschiede in der Färbung der Zellen auf zwei verschiedenen Faktoren beruhen: 1) Die charakteristischen Unterschiede in der Färbung im Lichte und in Dunkeln, 
wie sie sich in Eosin und in solchen Eosin-Methylenblaumischungen, in denen die Eosinkomponente stark tiberwiegt, sowie in Neutralrotlösungen zeigen, treten auf, wenn lebende Zellen in die Lösungen gebracht werden. Sind alle Zellen tot, so hören diese Unterschiede auf. Also das Licht beeinflußt die Färbung der Zellen dadurch, daß es die Zellen selbst verändert und sie so zu der Aufnahme gewisser Farbstoffe geeignet macht. Und es liegt die Annahme nahe - und hierfür werden später noch weitere Tatsachen angefuhrt werden -, daß die Zellen deshalb sich im Lichte anders färben als wie im Dunkeln, weil das Licht unter den gegebenen Versuchsbedingungen die Vitalität der Zellen beeinträchtigt. Viele der Zellen, die sich im Hellen und später auch im Dunkeln allmählich mit Eosin färben, sind tote Zellen. 2) Andre Unterschiede in der Färbung der Zellen beruhen hingegen auf entsprechenden Unterschieden in der Färbung der Lösungen. Dies gilt insbesondere für die Färbung der in Methylenblaulösungen und vielleicht auch in einigen andern Farbstofflösungen befindlichen Zellen. Dieser zweite Faktor bewirkt jedoch relativ nur geringe Unterschiede in der Färbung der Zellen; der unter 1 angefuhrte Bedingungskomplex ist viel wichtiger. Beide Faktoren können dadurch gegeneinander abgegrenzt werden, daß man die Zellen vor dem Einbringen in die Lösungen der Farbstoffe abtötet.

c) Wir sahen also, daß das Licht nur dann gewisse Unterschiede in der Färbung der Zellen hervorbringt, wenn es auf lebende Zellen wirken kann. Es folgt nun aus den Untersuchungen von Straub, v. Tappeiner und Edeferen, daß die Wirkung des Eosins im Lichte auf die Funktionen von Zellen oder auf die Tätigkeit von Fermenten an die Gegenwart des Sauerstoffs gebunden ist, also wahrscheinlich auf Oxydation beruht. Es wurde deshalb die Wirkung des Lichtes auf die Färbung der Zellen unter Bedingungen untersucht, in denen den gewöhnlichen Anschauungen zufolge die Oxydationsvorgänge in den Zellen verringert oder aufgehoben sind. Es wurde deshalb in erster Linie Zusatz von $\mathrm{KCN}$ zu den Farbstofflösungen versucht.

Insbesondere die Versuche von J. LoEB ${ }^{1}$ ) weisen darauf hin, daß Eier in Seewasser, dem $\mathrm{KCN}$ in gewissen Proportionen zugesetzt wurde, sich wie Eier verhalten, die sich in Seewasser befinden, aus dem der Sauerstoff durch Einleiten von Wasserstoff verdrängt wurde.

1) JAcques Loes, Versuche über den chemischen Charakter des Befruchtungsvorganges. Biochemische Zeitschr. Bd. I. 1906. 
Es wurde daher in verschiedenen Versuchen zu je $50 \mathrm{ccm}$ der Farbstofflösung in Seewasser $0,1,0,5$ und $4 \mathrm{ccm}$ einer $1 / 20 \%$ igen KCN-Lösung zugesetzt. Zusatz von $4 \mathrm{ccm}$ der KCN-Lösung verhindert die Entwicklung der Eier. Es ergab sich nun, daß Zusatz von KCN die Wirkung des Lichtes auf die Färbung der Zellen in den Methylenblau-Eosinmischungen in keiner Weise beeinträchtigt. Das gleiche gilt für Lösungen von Neutralrot nach Zusatz von 0,1 und $0,5 \mathrm{ccm}$ der KCN-Lösung (stärkere KCN-Lösungen konnten mit Neutralrot noch nicht untersucht werden). Es ergibt sich also aus diesen Versuchen, daß Beeinträchtigung der Oxydationsvorgänge der Zellen, wie sie wahrscheinlieh durch KCN bewirkt werden, nicht den Einfluß des Lichtes auf die Färbung der Zellen vermindert. Zusatz von $\mathrm{KCN}$ zu den Lösungen hat also nicht dieselben Wirkungen wie ein Erwärmen der Zellen auf $47^{\circ}$. Diese Beobachtungen stimmen überein mit den Ergebnissen von Joplbauer und v. Tappeiner ${ }^{1}$ ), die fanden, daß Zusatz ron KCN zu Invertin nicht die Einwirkung des Lichtes auf in Eosinlösungen befindliches Ferment verhindert.

d) Es wurden nun weiterhin Versuche angestellt, in denen der Sanerstoffgehalt der Farbstofflösungen vermehrt oder vermindert wurde, indem in die Schalen, welche die Lösungen und Eier enthielten, entweder Sauerstoff oder Wasserstoff' kontinuierlich eingeleitet wurde. Bei der gegebenen Versuchsanordnung ist es sehr unwahrscheinlich, daß völliger Mangel an Sauerstoff durch Einleiten von Wasserstoff bewirkt wurde; jedenfalls aber wurde hierdurch der Gehalt der Lösung an Sauerstoff sehr verringert.

Es ergab sich nun, daß Einleiten von Wasserstoff oder Sauerstoff die Einwirkung des Lichtes auf die Färbung der Zellen nicht merklich veränderte. Zu diesen Versuchen wurden Mischungen von Eosin und Methylenblau benutzt. Wie gewöhnlich war der Unterschied in der Färbung im Lichte und im Dunkel sehr deutlich. Weitere Versuche müssen zeigen, ob eine vollständige Verdrängung des Sauerstoffs aus den Lösungen die Unterschiede in der Färbung der Zellen im Lichte und im Dunkel aufhebt. Soweit stimmen die Ergebnisse dieser Versuche mit den über den Einfluß von KCN-Zusatz angestellten überein. Also der Einfluß des Lichtes änßert sich noch Zellen gegenüber, die unter den in $c$ und $d$ angegebenen $B e-$ dingungen gehalten werden. Es scheint also, daß das Licht die

1) A. Jodlbater und r. Tappeniner. Z̈ber die Beteiligung des Sauerstoffs bei der photodynamischen Wirkung fluorescierender Stoffe. Mïnchener Med. Wochenschrift. 28. Juni 1904. 
Zellen entweder viel eingreifender schädigt wie KCN-Zusatz oder Verminderung des Sauerstoffgehaltes der Flüssigkeit es tun, oder daß Licht Schädigungen bestimmter Art herbeiführt, die sich von den durch $\mathrm{KCN}$ - und 0-Armut bewirkten wesentlich unterscheiden.

e) Ebensowenig wie Zusatz von KCN oder Sauerstoffarmut die Einwirkung des Lichtes auf die Färbung der Zellen aufheben konnte, vermehrte oder verminderte Zusati von Alkali $\left(1-2 \mathrm{ccm} \frac{\mathrm{N}}{100} \mathrm{NaOH}\right.$ zu 50 cem Lösung des Farbstoffs in Seewasser) zu Eosin-Methylenblaugemischen den Einfluß des Lichtes auf die Färbung der Zellen.

f). Es war nun noch die Möglichkeit zu berïcksichtigen, daß unter dem Einfluß des Lichtes in einem Gemisch von Eosin und Methylenblau Veränderungen stattfinden, welche die Giftigkeit dieses Gemisches für die Zellen erhöhen, und daß infolge dieser erhöhten Giftigkeit der Lösung im Lichte die Zellen sich stärker mit Eosin färben. In diesem Fall würde also das Licht primär auf die Farbstoffmischung wirken und nur indirekt auf die Zellen. Es müßte dann möglich sein, dieselben Farbewirkungen im Dunkeln zu erhalten, falls Farbmischungen verwandt würden, die vorher längere Zeit im Lichte standen. Es zeigte sich nun, daB dies nicht der Fall ist. Mischungen von Eosin und Methylenblau, die 6 Stunden dem Lichte ausgesetzt waren und welche sodann nach dem Zusatz der Eier im Dunkeln gehalten wurden, waren nicht giftiger für die Eier als frisch bereitete Mischungen derselben Farbstoffe, die in gleicher Weise einen Zusatz von Eiern erhielten und im Dunkeln gehalten wurden. Es schienen im Gegenteil die vorher dem Lichte ausgesetzten Mischungen weniger giftig auf die Eier zt wirken wie die Kontrolllösungen. Jedenfalls war die Färbung die gleiche wie bei den Eiern, welche in den gewöhnlichen Eosin-Methylenblaumischungen im Dunkeln gehalten wurden.

g) Es ergibt sich also aus allen diesen Versuchen, daß die Unterschiede in der Färbung der Eier im Lichte und im Dunkeln zum Teil auf einer direkten schädlichen Einwirkung des Lichtes auf die in den Farblösungen befindlichen Eier beruht. Nach Abtöten der Zellen nehmen die Zellen dieselbe Färbung im Dunkeln an, wie sonst nur unter dem Einfluß des Lichtes. Andre Unterschiede in der Färbung der Zellen im Lichte und im Dunkel sind nur passir und beruhen darauf, daß die Zellen die Farbe der Lösung annehmen. Es fragt sich nun noch, ob der Tod der Zellen nötig ist, damit die für das Licht charakteristische Färbung auftritt, oder aber Schädigungen 
der Zelle, die das Leben der Eier ermöglichen, hierfür gentigen. Wir sahen, daß Zusatz von KCN und Verminderung des Sauerstoffs nicht gentigen, um die Unterschiede in der Färbung im Dunkeln und im Lichte aufzuheben.

h) Daß solche Zellen, welche stark geschädigt sind, die für das Licht charakteristischen Färbungen annehmen, ergibt sich auch aus einer Anzahl ron Einzelbeobachtungen tiber die Art and Lokalisation der Färbung in den einzelnen Lösungen. Mit Neutralrot färben sich die gesunden Zellen anders als die toten oder absterbenden Zellen. Schwimmende Blastulae oder Gastrulae färben sich tief braunrot, zuweilen mit einer Nuance violett. Die Färbung ist meịst so intensir, daß feinere Strukturverhältnisse nicht erkennbar sind. Woblerhaltene ungeteilte Eier färben sich hingegen kirschrot; am wenigsten sind gewöhnlich solche Eier gefärbt, die in mehrere Segmente geteilt sind. Tritt granuläre oder hyaline Substanz aus dem segmentierten oder unsegmentierten Ei aus, so färbt diese sich relativ am wenigsten mit Neutralrot oder Methylenblau, am stärksten hingegen mit Eosin. Zerfällt eine Blastula oder Gastrula teilweise, d. h. werden Zellen ans dem Verband der normalen Zellen ausgestoßen, so sammeln sich diese häufig unter der Membran an. Die Blastula oder Gastrula bewegt sich dann mit Hilfe ihrer Cilien innerbalb der Masse ausgestoBener, absterbender Zellen. Diese ausgestoßenen granulären Massen (ausgestoßene Zellen) färben sich nun ebenfalls am wenigsten mit Neutralrot oder Methylenblau; sie färben sich gewöhnlich stark mit Eosin. Doch können kurze Zeit nach ihrer Ausstoßung diese Zellen noch Neutralrotfärbung annehmen; sie verlieren diese aber gewöhnlich bald. Es kommt unter den in diesen Versuchen gegebenen Bedingungen nicht selten vor, daB nur ein Teil der Segmente sich zur Blastula oder Gastrula weiter entwickelt, während ein andrer Teil der Segmente desselben Eies auf der früheren Entwicklungsstufe verharrt und allmählich abstirbt. Wir finden dann unter derselben Membran eine kleine bewegliche Blastula oder Gastrula, die sich stark mit Nentralrot färbt, und daneben Segmente, die entweder leicht gelbbraun oder ungefärbt sind, oder aber sich mit Eosin färben. Am ehesten scheinen diejenigen segmentierten Eier sich mit Eosin zu färben, deren Membran weit abgehoben ist. Bei der Abhebung der Membran handelt es sich vielleicht um der Plasmolyse von Pflanzenzellen ähnliche Vorgänge; sie betrifft wahrscheinlich stark geschädigte Eier.

Allen diesen Beobachtungen gemeinsam ist die Tatsache, daß besonders geschädigte absterbende oder tote Zellen oder Zellteile 
sich mit Neutralrot am wenigsten, mit Eosin relativ am stärksten färben.

Ferner ist allen diesen Befunden gemeinsam die Tatsache, daß absterbende oder tote Zellteile oder Zellen die für Licht charakteristische Färbung annehmen, während die lebenden Zellen oder Zellteile sich den Farblösungen gegeniiber wie die im Dunkeln gehaltenen Zellen verhalten.

In ähnlicher Weise ist wahrscheinlich eine Beobachtung zu erklären, die Herr SHIPPEN machte, als er im Sommer 1905 auf meine Veranlassung die Entwicklung der Eier versehiedener Wirbellosen in Eosinlösungen im Lichte und im Dunkeln untersuchte. Er fand, daB auch schwimmende Blastulae und Gastrulae sich zum Teil mit Eosin färben können. Es zeigt sich nun bei genauerer mikroskopischer Untersuchung, daß nicht die gesunden Zellen der äußeren Zellschicht diese Färbung annehmen, sondern es handelt sich hierbei um eine Färbung von Zellen oder Zellfragmenten, die nach dem Innern abgestoßen und die entweder tot oder stark geschädigt sind.

Diese Tatsache ist nun auch noch deswegen von besonderem Interesse, weil hierbei, wie es scheint, Eosin eine Schicht gesunder Zellen durchdringt, um zu den im Inneren gelagerten Zellen zu gelangen und diese $z u$ färben. $\mathrm{Es}$ ist nicht wahrscheinlich, daß die Zellen bereits zu einer Zeit die Eosinfarbe annehmen, wenn sie sich noch in dem Verband der gesunden Zellen befinden, da es mir nicht möglich war, in dieser Lage eosingefärbte Zellen zu sehen. Auf welche Weise diese Färbung stattfindet, bleibt noch zu untersuchen. Vielleicht kann folgende Beobachtung, die ich allerdings bisher nur einmal machte, zur richtigen Erklärung führen: Zerdruickt man ein anscheinend normales und ungefärbtes $\mathrm{Ei}$, das sich in einer schwachen Eosinlösung findet, so fließt Zellsaft aus. Dieser kann nun Eosin enthalten, obwohl die Zellgranula und die die Granula verbindende zähflïssige, fadenbildende Masse ungefärbt sind. Es wäre möglich, daß Eosin in lebende Zellen eindringt, ohne die strukturellen Elemente zu färben. Eine solche Färbung findet erst beim Absterben statt. $O b$ diese Erklärungsweise zutrifft, miissen weitere Untersuchungen zeigen.

Also in lebenden Blastulae oder Gastrulae fürbt sich nur die ins Innere abgestoßene Lage von Zellen. Stirbt die Blastula oder Gastrula ab, so beginnt umgekehrt die Eosinfärbung außen und dringt allmählich nach innen vor.

Mit Neutralrot fürben sich die gesunden Zellen lebender Blastulae 
und Gastrulae. In Methylenblau müssen wir zwei Arten von Färbung unterscheiden. 1) Es kommt eine diffuse Blaufärbung der außeren gesunden Zellen der Blastula ror. 2) Zuweilen findet sich eine fleckweise tiefere Methylenblaufärbung; diese scheint hauptsächlich gewisse Bezirke der nach innen abgestoßenen Zellen zu betreffen; es ist aber wahrscheinlich, daß auch Zellen der äußeren Schicht sich ähnlich färben können. Bei dieser zweiten Art der Fürbung scheint es sich um eine Färbung granulärer Massen zu handeln.

Es bleibt nun noch zu untersuchen, ob der entwicklungshemmende Einfluß des Lichtes und der die Fïrbung bestinımende immer parallel gehen. Gewisse Beobachtungen deuten darauf hin, daß dies nicht der Fall zu sein braucht, doch mitssen darüber noch weitere Versuche angestellt werden.

Es bleibt ferner noch zu untersuchen, ob das Licht die Entwicklung von Zellen hemmt, die in Seewasser ohne Beimischung von Farbstoffen gehalten werden. Es könnte sich hierbei nur um relativ geringfügige Unterschiede handeln, die um so schwerer festzustellen sind, als Fäulnisvorg:̈nge als ein komplizierender Faktor eingreifen können. Doch soll die Möglichkeit einer solchen Einwirkung des Lichtes nicht bestriten werden, insbesondere da LEO $\left.{ }^{1}\right)$ fand, daß gewisse oxydable Stoffe im Seewasser unter dem Einfluß des Lichtes stärker verändert werden, als die gleichen unter sonst gleichen Bedingungen, aber in destilliertem Wasser anstatt in Seewasser gehaltenen Stoffe.

\section{Zusammenfassung.}

1) In Lösungen von Farbstoffen (Neutralrot, Eosin, Methylenblan und andre) furben sich Zellen (Eier von Asterias) verschieden, je nachdem Zellen und Lösungen dem Lichte ausgesetzt sind oder in Dunkeln gehalten werden. Lösungen von Eosin und Farbmischungen, die Eosin enthalten, haben im Lichte eine stärker hemmende Wirkung auf die Entwicklung der Eier als in Dunkeln. Dasselbe gilt wahrscheinlich für Neutralrot.

2) Kombination eines sauren und eines basischen Farbstoff's (Eosin und Methylenblau) verstärkt den Unterschied in der Fürbung. der Zellen im Lichte und im Dunkeln bedeutend, hauptsïchlich dadurch, daß schon ein geringer Zusatz ron Methylenblau genügt, um

1: I. Leo, Über dic Beeinflussung ded Sommenlichtwirkung durch Meerwasser. Deutsche med. Wochenschrift. Nr. 52. 1904. 
die für das Licht charakteristische Färbung bedeutend zu verstärken. Wahrscheinlich gilt dasselbe für andre ähnliche Kombinationen $\{\mathrm{z}$. B. Neutralrot-Eosin). Eine neutralisierende Wirkung von basischen und sauren Farbstoffen besteht daher nicht.

Diese verstärkende Wirkung der Kombination von Methylenblau und Eosin beruht nicht anf einer durch das Licht in der Farbstoffmischung hervorgerufenen Veränderung, da nach vorheriger Lichtexposition der Farbstoffmischung diese die Zellen im Dunkeln nicht anders fürbt als vorher nicht exponierte Lösungen.

Bei Kombination zweier basischer Farbstoffe (Methylenblau-Neutralrot) vertreten sich Methylenblau und Neutralrot im Dunkeln, während im Lichte die Zellen eine Mischfarbe annehmen.

3) Der Unterschied in der Färbung der Zellen im Lichte und im Dunkeln beruht zum mindesten auf zwei verschiedenen Einwirkungen des Lichtes. a) Das Licht bewirkt primär Veränderungen in den Zellen und der Unterschied in der Färbung im Lichte und im Dunkeln beruht anf den in den Zellen durch das Licht hervorgerufenen Veränderungen (Färbungen mit Eosin, Neutralrot und mit gewissen Mischungen von Eosin und Methylenblau und von Eosin und Neutralrot). b) Das Licht verändert primär die Farbstofflösungen und die Färbung der Zellen ist eine passive, der Farbstofflösung entsprechende (Methylenblau und solche Methylenblan-Eosinmischungen, in denen viel Methylenblau vorhanden ist; vielleicht auch Lösungen von Hämatoxylin).

Weiterhin hängt die Färbung der Zellen sowohl im Lichte wie im Dunkeln von den Proportionen ab, in denen beide Farbstoffe in der Mischung vorhanden sind.

4) Abtöten der Zellen (durch Wärme) ermöglicht die Unterscheidung der unter $a$ und $b$ genannten, die Zellfärbung bedingenden Faktoren; nach der Abtötung der Zellen sind die durch direkte Einwirkung des Lichtes auf die Zellen bewirkten Unterschiede in der Färbung aufgehoben, während die passiven durch die Veränderung der Farbstoff lösungen bedingten Unterschiede noch vorhanden sind.

5) Mittel, welche wahrscheinlich die oxydativen Prozesse der Zellen herabsetzen (Zusatz von KCN, Durchleiten von Wasserstoff durch die Lösungen), und konstantes Durchleiten von Sauerstoff durch die Lösungen verändern die Unterschiede in der Färbung der Zellen im Lichte und im Dunkeln nicht merklich; es ist daher nicht wahrscheinlich, daß das licht die Zellfärbung dadurch beeinflußt, daß es die oxydativen Prozesse in den Zellen steigert. Auch der Zusatz von 
378 Leo Loeb, Über den Einfluß des Lichtes auf die Färbung usw.

Alkali zu den Farbstofflösungen ist ohne Einfluß auf die Färbung der Zellen im Lichte oder im Dunkeln.

6) Eine Reihe von Beobachtungen über das Verhalten versehiedener Eier und Larven in den verschiedenen Farbstofflösungen macht es wahrscheinlich, daß der Einfluß des Lichtes zum Teil auf einer Schädigung oder Abtötung von Zellen berubt und daß die Unterschiede in der Färbung sekundär sind. Insbesondere sei auf die verschiedene Färbung schwimmender Brastulae und Gastrulae mit Losin einerseits und mit Neutralrot und Methylenblau anderseits hingewiesen. Mit den beiden letztgenannten Farbstoffen, insbesondere mit Neutralrot, fürben sich die äußeren, gesunden Zelllagen lebender Zellen, während mit Eosin sich die nach innen oder außen abgestoßenen Zellen der Blastulae oder Gastrulae färben. 